\title{
Self-Diffusion of Entangled Linear and Circular DNA Molecules: Dependence on Length and Concentration
}

\author{
Rae M. Robertson and Douglas E. Smith* \\ Department of Physics, University of California, San Diego, Mail Code 0379, 9500 Gilman Drive, \\ La Jolla, California 92093
}

Received January 8, 2007; Revised Manuscript Received March 1, 2007

\begin{abstract}
Self-diffusion coefficients $(D)$ of DNA molecules of varying length and concentration were measured by tracking the Brownian motion of individual fluorescently labeled tracer molecules. Four possible cases were examined: linear tracer molecules surrounded by linear molecules $(L-L)$, circular tracers surrounded by linears $(C-L)$, linear tracers surrounded by circles $(L-C)$, and circles surrounded by circles $(C-C)$. With 6 and 11 kilobasepair (kbp) DNA $D$ was largely insensitive to topology and varied consistent with Rouse scaling $\left(D \sim L^{-1} C^{-0.5}\right.$ ). In contrast, with 25 and $45 \mathrm{kbp}$ DNA topology had a strong influence. At $1 \mathrm{mg} / \mathrm{mL}$ we found $D_{C-C}>D_{L-C} \gg D_{L-L} \gg D_{C-L}$. In the $L-L, L-C$, and $C-C$ cases a crossover from scaling consistent with the Rouse model to scaling consistent with the reptation model $\left(D \sim L^{-2} C^{-1.75}\right)$ was observed at $\sim 6$ times the molecular overlap concentration. In contrast, $D_{C-L}$ decreased much more steeply with concentration, indicating that a process much slower than reptation governs that case.
\end{abstract}

\section{Introduction}

The physical behavior of entangled polymer solutions has been the subject of a plethora of theoretical and experimental studies. Entangled linear polymer solutions have been thoroughly investigated, ${ }^{1}$ and molecular diffusion and linear viscoelastic response in such systems have been found to be well described by the reptation model of deGennes ${ }^{2}$ and Doi and Edwards. ${ }^{3}$ The key assumption of this model is that on short time scales any given "tracer" molecule is confined by entanglements with the surrounding "matrix" molecules to move within a tubelike region parallel to its own contour. Such tubelike motion has been directly imaged by fluorescence microscopy. 4,5 In this regime, the predicted ${ }^{3}$ scaling of the self-diffusion coefficient $(D)$ with molecular length $(L)$ and concentration $(C)$ in good solvent conditions is $D \sim L^{-2} C^{-1.75}$. Below a certain concentration $\left(C_{\mathrm{e}}\right)$ or length $\left(L_{\mathrm{e}}\right)$ (but above the molecular overlap concentration, calculated as $C^{*} \cong(4 / 3) \pi M / N_{\mathrm{A}} R_{\mathrm{G}}{ }^{3}$ where $M$ is molecular weight, $N_{\mathrm{A}}$ is Avogadro's number, and $R_{\mathrm{G}}$ is the radius of gyration ${ }^{3}$ ) entanglement effects should no longer dominate, and $D$ is expected to follow the predictions of the Rouse model, ${ }^{3,6} D \sim L^{-1} C^{-0.5}$. Below $C^{*}$, the Rouse model is no longer valid because hydrodynamic interactions become important, and $D$ is predicted by the Zimm model to be independent of $C$ and to scale as $D \sim L^{-0.588}$.

While the predicted scaling laws for both the Rouse and reptation regimes have been confirmed for linear polymers, 7,8 fewer studies have examined the crossover region. Most have used polymer melts, where the concentration is not variable, to achieve high degrees of entanglement. Further, there is no clear consensus on the molecular length scales and concentrations over which this shift from the Rouse to the reptation regime occurs, ${ }^{9,10}$ and no reliable way to calculate $L_{\mathrm{e}}$ and $C_{\mathrm{e}}$ on the basis of molecular theory has been proposed.

Circular polymers (also referred to as cyclic or ring polymers) have been the subject of far fewer studies. As they have no ends by which to escape tubelike constraints, they seemingly could not undergo normal reptation and are thus an intriguing case. Furthermore, DNA is naturally found in both linear and circular forms, making this a case of practical interest. Constraint release, the shifting of the matrix chains constraining the tracer chain motion, has been predicted to play an important role in circular polymer diffusion, especially when the chains forming the entanglements are linear. ${ }^{11}$ Klein proposed three possible conformations for circular tracers in melts of linear polymers ( $C-L$ melts): (A) the circle is pinned or threaded by the surrounding chains, (B) it is unpinned but ramified (large loops are present), or (C) it is unpinned and linear (no large loops). ${ }^{11}$ The probability of (B) and (C) to occur was predicted to be an exponentially decaying function of tracer chain length, and the process by which (C) diffuses is predicted to be similar to that for reptation of a linear chain of length $L / 2$. Disregarding case (B), which would have a lower self-diffusion coefficient than (C), the predicted contribution from the unpinned cases for a circular tracer chain is $D_{\mathrm{U}} \sim D_{\text {rep }}(L / 2) \mathrm{e}^{-\beta L}$, where $\beta$ is a constant. Reptation is not possible in case (A), and the proposed mechanism is constraint release.

Graessley predicted that a polymer diffusing by constraint release would obey $D_{\mathrm{CR}} \sim L^{-1} L_{\mathrm{m}}{ }^{-3}$, where $L$ is the tracer chain length and $L_{\mathrm{m}}$ is the length of the matrix chains. ${ }^{12}$ Klein modified this prediction, suggesting $D_{\mathrm{CR}} \sim L^{-1} L_{\mathrm{m}}{ }^{-5 / 2}$, and further predicted the net self-diffusion coefficient for the $C-L$ case to be $D_{C-L}=D_{\mathrm{U}}+D_{\mathrm{CR}}$. For small tracer chains the probability for (A) to occur was predicted to be extremely low, such that $D_{C-L} \sim D_{\mathrm{U}}$. As $L$ is increased, $D_{\mathrm{CR}}$ is predicted to dominate. In addition, Mills considered a circle threaded only once by a linear chain, ${ }^{13}$ predicting $D_{\mathrm{P} 1} \sim L^{-1} L_{\mathrm{m}}{ }^{-1}$ for this confirmation. The $L-C$ and $C-C$ cases were not addressed in these theories, although Klein conjectured that constraint release would be negligible in the $C-C$ case and that reptation would dominate even at long chain lengths. ${ }^{11}$ What molecular geometries would allow for such behavior is unclear.

Several simulations of $C-C$ and $L-L$ polymer melts have been carried out and $D \sim L^{-v}$ was reported with $v$ ranging from 0.9 to 2 , consistent with Rouse and reptation predictions. Three studies predicted $D_{C-C}>D_{L-L}$ for all lengths examined, ${ }^{14-16}$ whereas one predicted a crossover from $D_{C-C}<D_{L-L}$ to $D_{C-C}$ $>D_{L-L}$ above a certain length. ${ }^{17,18}$ One study also predicted that $L_{\mathrm{e}}$ would be $\sim 2-5$ times higher for the $C-C$ case than for 
the $L-L$ case. ${ }^{15}$ However, no theories are available for direct comparison, as none have considered the concentration dependence of $D$ for solutions of circular polymers or combinations of circular and linear polymers.

The majority of previous experiments with circular polymers compared $D_{C-C}$ to $D_{L-L}$ for polymer melts. In disagreement with the simulations, three experimental studies found $D_{L-L}>D_{C-C}$ for all lengths examined, ${ }^{19-21}$ and two reported that $L_{\mathrm{e}}$ was roughly the same for $L-L$ and $C-C .^{22,23}$ Scaling exponents of $v=1-2.34$ were reported.

Three experimental studies have examined $C-L$ melts. One reported $D_{C-L} \sim L^{-0.83}$, concluding that the length range used was too low to study entanglement effects. ${ }^{21}$ A second found $D_{C-C} \cong D_{C-L}$, with values in accord with the $L-L$ case, concluding that both systems diffused by reptation. ${ }^{24}$ Threading or pinning of the tracer was thought to be unlikely in the $C-L$ case with the short length used. The third study found $D_{C-L}>$ $D_{L-L}$ crossing over to $D_{L-L}>D_{C-L}$ as $L$ was increased. ${ }^{13}$ These $D_{C-L}$ values lie between those predicted by reptation and constraint release models. For the longest matrix length $D_{C-L}$ $\sim L^{-3.2} L_{\mathrm{m}}{ }^{-\alpha}$, with $\alpha$ ranging from 0 to 1.6 , was reported, in contradiction with the prediction of $D_{\mathrm{CR}} \sim L^{-1} L_{\mathrm{m}}{ }^{-3}$ for simple constraint release $\mathrm{e}^{12}$ but in accord with the once-threaded circle model. ${ }^{13}$

Only one experimental study has compared the $L-C$ and $L-L$ cases, finding $D_{L-C} \cong D_{L-L}$ for all lengths studied, in qualitative accord with the predicted result for a linear tracer undergoing reptation and constraint release in a linear matrix. ${ }^{24}$ Also, only one experimental study investigated concentration dependence with circular polymer solutions, ${ }^{25}$ finding a similar trend for $C-C$ and $L-L$, but with $D_{C-C}>D_{L-L}$. Although a good solvent was used in this experiment, $D_{C-C} \sim D_{L-L} \sim C^{-3.0}$ was unexpectedly observed, which is the scaling predicted for a $\Theta$ solvent (vs $D_{L-L} \sim C^{-1.75}$ for good solvents).

DNA, in addition to being of specific interest in biophysics, is an excellent model system for studying polymer physics. ${ }^{26-28}$ Recent measurements have shown that the rheological behavior of DNA is strikingly similar to that of synthetic polymers. ${ }^{28,29}$ Homogeneous samples can be produced by DNA replication, ${ }^{30}$ and both linear and relaxed circular forms can be prepared. ${ }^{30,31}$ Fluorescence video microscopy enables direct visualization of single polymer dynamics. ${ }^{4,27,28}$ Previously, we studied four linear DNA constructs with a length range 6.6-48.5 kilobasepairs (kbp) diffusing in solutions of linear $\lambda$-DNA (48.5 kbp).$^{5}$ Results with 48.5 or $23.1 \mathrm{kbp}$ tracer molecules were consistent with $D$ $\sim C^{-1.75}$ for concentrations of $0.5-0.8 \mathrm{mg} / \mathrm{mL}$. At $0.63 \mathrm{mg} /$ $\mathrm{mL} D \sim L^{-1.8 \pm 0.1}$ was found.

Here, we comprehensively examine how the self-diffusion coefficient of a tracer DNA molecule depends on concentration, length, and topology of both the tracer chain and the matrix chains. For the first time we examine all four possible topological cases: $L-L, C-L, C-C$, and $C-L$. For each case four different DNA lengths and 10 different concentrations were studied, chosen to span the range from semidilute to concentrated (entangled) regimes. $D$ values in the dilute limit for these molecules were reported previously. ${ }^{31}$

\section{Materials and Methods}

Double-stranded DNA molecules of 5.9, 11.1, 25, and $45 \mathrm{kbp}$ ( $\sim 39-300$ persistence lengths, also see Table 1 in the Supporting Information) were prepared by replication of cloned plasmid and fosmid constructs in Escherichia coli, as described previously. ${ }^{30}$ The restriction enzymes BamHI and ApaI were used to prepare the linear form, and Topoisomerase I was used to prepare the relaxed circular form. ${ }^{31}$ Tracer molecules were labeled with

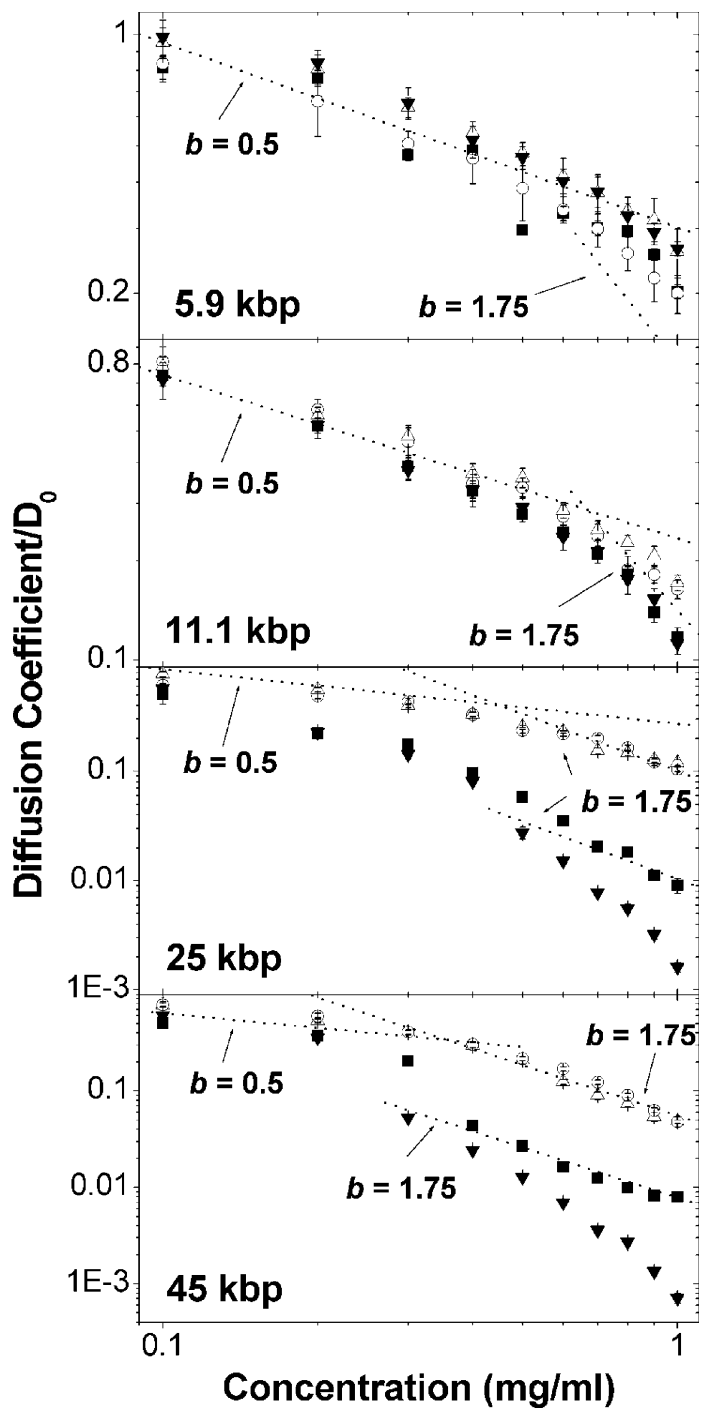

Figure 1. Self-diffusion rates vs concentration plotted for each of the four molecular lengths. The diffusion coefficients $(D)$ at each concentration are normalized by the values in the limit of zero concentration $\left(D_{0}\right)$. The different point styles indicate the four different topological cases: circular molecules in a circular matrix $(C-C)$ (open circles), linear molecules in a circular matrix $(L-C)$ (open triangles), circular molecules in a linear matrix $(C-L)$ (filled triangles), and linear molecules in a linear matrix $(L-L)$ (filled squares). The dotted lines indicate the scaling laws $D \sim C^{-b}$ predicted by the Rouse $(b=0.5)$ and reptation $(b=1.75)$ models for linear molecules.

YOYO-I (Molecular Probes) (dye molecule to base pair ratio of 1:6) and imaged with a custom-built epiflourescence microscope, as described previously. ${ }^{30,31}$ The samples were mixed with unstained DNA in an aqueous buffer solution (10 mM Tris- $\mathrm{HCl}(\mathrm{pH} 8)$, $1 \mathrm{mM}$ EDTA, $10 \mathrm{mM} \mathrm{NaCl}$ ) by slowly pipetting $\sim 20$ times with a wide-bore tip, which has been shown to yield a homogeneous solution. ${ }^{29}$ Oxygen scavenging and antioxidant reagents $(4 \%(\mathrm{v} / \mathrm{v})$ $\beta$-mercaptoethanol, $30 \%$ (w/v) glucose, $10 \mu \mathrm{g} / \mathrm{mL}$ glucose oxidase, and $120 \mu \mathrm{g} / \mathrm{mL}$ catalase) were added to reduce photobleaching. Diffusion coefficients $(D)$ were determined by tracking of Brownian motion, over a length scale of $\sim 2-10 R_{\mathrm{G}}$, as described previously. ${ }^{5,31,33}$ Briefly, the center-of-mass $(x, y)$ coordinates of $>1000$ paths of $>20$ different molecules were tracked, and the Einstein relationship $\left\langle x^{2}\right\rangle=\left\langle y^{2}\right\rangle=2 D t$ was used to determine the diffusion coefficient.

\section{Results}

Concentration Dependence. The measured dependences of $D$ on concentration are shown in Figures 1 and 2. First we compare the cases with circular matrix molecules. $D_{C-C}>D_{L-C}$ 
was observed for all concentrations and lengths. These two cases have not been directly compared in any previous study with any type of polymer. When the $D$ values were normalized by their corresponding values at infinite dilution, ${ }^{31}$ they approximately collapsed onto a single curve. With the shortest length $(5.9 \mathrm{kbp}) D \sim C^{-b}$ was observed, with $b_{C-C}=0.60 \pm$ 0.04 and $b_{L-C}=0.56 \pm 0.04$, only slightly higher than $b=$ 0.5 predicted by the Rouse model. With the three longer molecules $b \cong 0.5$ was observed at low concentration, but a significant increase in $b$ was noted starting at $\sim 0.7,0.5$, and $\sim 0.3 \mathrm{mg} / \mathrm{mL}$ for $L=11.1,25$, and $45 \mathrm{kbp}$, respectively. The prediction $b=1.75$ of the reptation model is approximately (tangentially) reached at $\sim 0.9, \sim 0.7$, and $0.4 \mathrm{mg} / \mathrm{mL}$ for $L=$ $11.1,25$, and $45 \mathrm{kbp}$, respectively, with both tracer topologies, indicating the onset of entanglement. Thus, $C_{\mathrm{e}}$ decreases with increasing $L$ in both cases. Present theories have not considered these cases, so no comparison with theory is possible. We note that our finding differs from the aforementioned finding by pulsed-gradient NMR of $D_{C-C} \sim D_{L-L} \sim C^{-3.0}$ for poly(ethylene oxide) solutions. ${ }^{25}$

Next we compare the cases where the matrix molecules are linear (Figures 1 and 2). While the trends in the $L-L$ and $C-L$ cases were similar to those observed in the $L-C$ and $C-C$ cases with 5.9 and $11.1 \mathrm{kbp}$ molecules, dramatically different behavior was observed with the 25 and $45 \mathrm{kbp}$ molecules. We observed a crossover from $D_{C-L}>D_{L-L}$ to $D_{L-L}>D_{C-L}$ at a concentration of $\sim 4 C^{*}$. $D_{L-L}$ reached $b \cong 1.75$ at roughly the same concentration as $D_{L-C}$ and $D_{C-C}$, leading to the relationship $C_{\mathrm{e}}$ $\cong 6 C^{*}$ for the $C-C, L-C$, and $L-L$ systems. The finding that $C_{\mathrm{e}}$ is independent of topology is in qualitative accord with the topology-independent $L_{\mathrm{e}}$ found in rheology measurements on $L-L$ and $C-C$ polysterene and poly(dimethylsiloxane) melts. ${ }^{22,23}$

Our results illustrate that the topologies of both the tracer and matrix molecules can have a strong effect on diffusion. At $1 \mathrm{mg} / \mathrm{mL} D_{C-L}$ was $\sim 10$-fold smaller than $D_{L-L}$ and $\sim 100$-fold smaller than $D_{C-C}$. Our findings are qualitatively consistent with Klein's prediction for $C-L$ melts that as the entanglement density is increased, constraint release, which is a much slower mode of diffusion than reptation, would dominate. Our findings are also consistent with Klein's conjecture that constraint release would be negligible for $C-C$ melts and that reptation is the dominant mechanism. We note that circular polymers cannot diffuse by conventional reptation as they have no ends. However, in Klein's model (conformation (C)) the circles were envisioned to adopt a collapsed form with the two antiparallel halves of the circle lying tangent to each other, and the reptation was envisioned to be analogous to that of a linear polymer of half the length. Our result of $D_{C-C}>$ $D_{L-L}$ agrees qualitatively with the findings of the studies of poly(ethylene oxide) solutions by pulsed-gradient NMR. ${ }^{25}$ However, we find that $D_{C-C} / D_{L-L}$ increases from $\sim 2$ to $\sim 10$ as the scaling changes from Rouse-like to reptation-like, while this ratio was found to have a constant value of $\sim 1.5$ in the NMR studies. One possible explanation for this discrepancy could be the difference in the relative concentrations examined. This NMR study probed concentrations up to $\sim 6 C^{*}$ while our highest concentration was $\sim 12 C^{*}$ for the $45 \mathrm{kbp}$ molecule.

Length Dependence. The length dependences are shown in Figure 3. At low concentrations we find $D_{C-C}>D_{C-L}>D_{L-C}$ $>D_{L-L}$ for all lengths. As the concentration was increased, this relationship shifted to $D_{C-C}>D_{L-C}>D_{L-L}>D_{C-L}$ at $\sim 4 C^{*}$ with the 25 and $45 \mathrm{kbp}$ molecules. This is in agreement with the prediction $D_{C-C}>D_{L-L}$ of three Monte Carlo simulation studies $^{14-16}$ but in disagreement with one Monte Carlo study

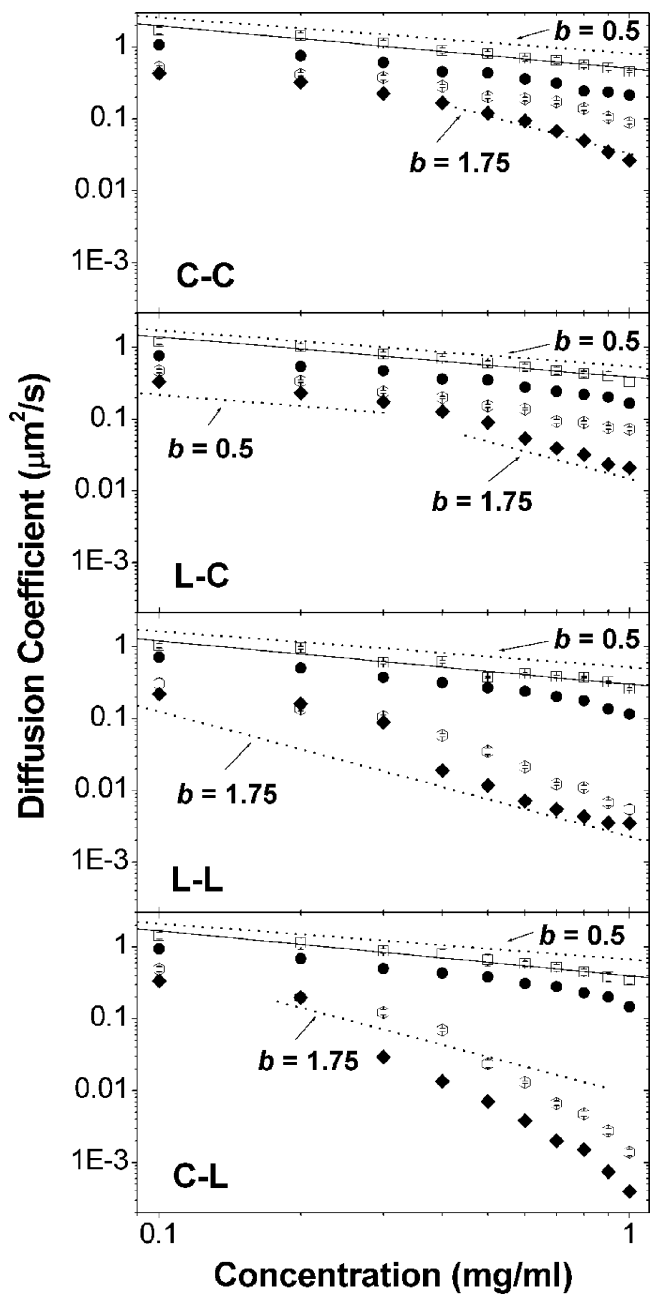

Figure 2. Self-diffusion rates vs concentration plotted for each of the four topological cases. These data are from the same set of measurements as in Figure 1. The different point styles indicate the four different construct lengths: $5.9 \mathrm{kbp}$ (open squares), $11.1 \mathrm{kbp}$ (filled circles), $25 \mathrm{kbp}$ (open hexagons), and $45 \mathrm{kbp}$ (filled diamonds). The solid lines are power law fits to the $5.9 \mathrm{kbp}$ data giving scaling exponents of $b_{C-C}=0.60 \pm 0.04, b_{L-C}=0.56 \pm 0.04, b_{L-L}=0.61 \pm 0.06$, and $b_{C-L}=0.63 \pm 0.05$. The dotted lines are as in Figure 1 .

and findings on alkanes and PDMS by pulsed-gradient spinecho NMR. ${ }^{17,19-21}$ While our results can be compared to and are in partial agreement with these NMR studies, it is important to note that there are key differences between these studies and ours. First, they examined melts with no solvent, in contrast to our study where the volume fraction of DNA in solution is $\sim 10^{-3}$. Further we examine different diffusion lengths. In the previous studies, the length scale of diffusion during the duration of the experiments ranged from $\sim 4$ to $\sim 1000$ times the contour lengths of the polymers used. This length scale decreased as the molecular length increased as the larger polymers diffused much more slowly. Here, the diffusion lengths ranged from $\sim 2$ times the contour length $\left(\sim 10 R_{\mathrm{G}}\right)$ for the $6 \mathrm{kbp}$ molecule to $\sim 1 / 10$ the contour length $\left(\sim 2 R_{\mathrm{G}}\right)$ for the $45 \mathrm{kbp}$ molecule.

Here, in all cases the two shorter constructs showed a scaling exponent between $\sim 0.5$ and 1 , showing that these solutions were in the dilute to semidilute regimes (predicted exponents of 0.588 and 1 , respectively). This behavior is similar to that found by NMR in the $C-C$ and $C-L$ cases $\left(v_{C-C} \cong 1\right.$ and $v_{C-L}$ $\cong 0.83){ }^{19-21}$ Scaling exponents with the 25 and $45 \mathrm{kbp}$ molecules, in contrast, ranged from $<1$ to $\sim 2$ at high concentrations for all the topological cases. Similar behavior was reported previously for $C-C$ and $L-L$ melts in three simulation studies 


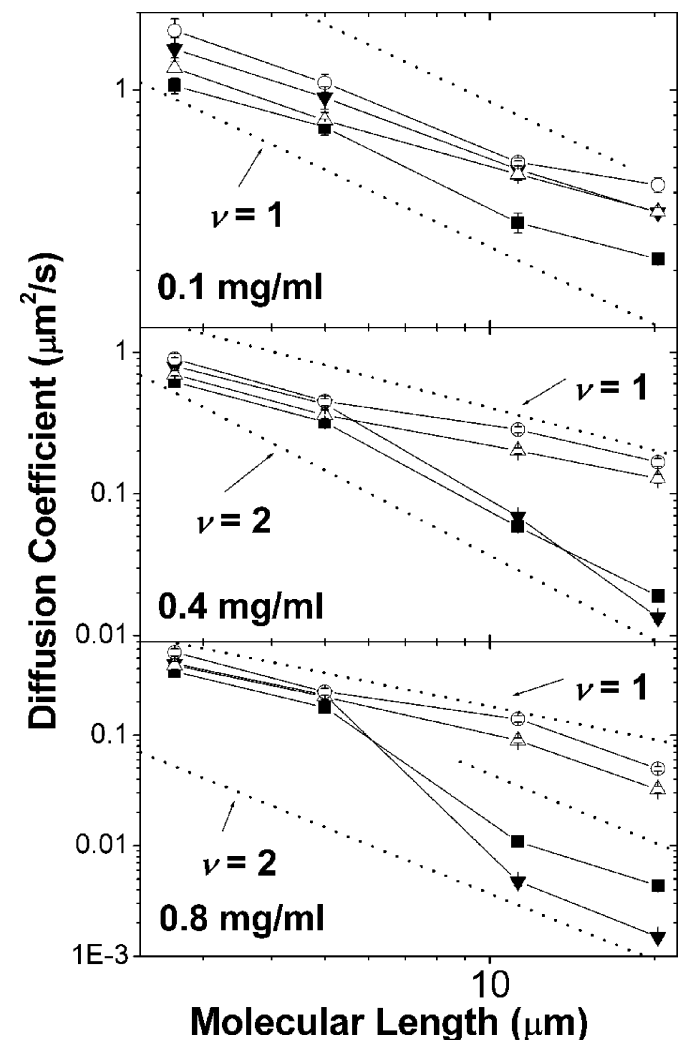

Figure 3. Self-diffusion coefficients vs DNA length plotted for three different concentrations. These data are also from the same set of measurements as in Figure 1. The different point styles indicate the four different topological cases: $C-C$ (open circles), $L-C$ (open triangles), $L-L$ (filled squares), and $C-L$ (filled triangles). The dotted lines indicate the scaling laws $D \sim L^{-v}$ predicted by the Rouse $(v=1)$ and reptation $(v=2)$ models for linear molecules.

and one of the NMR studies. ${ }^{14,16,17,21}$ A shift from Rouse $(v \cong 1)$ to reptation $(v \cong 2)$ scaling with these two constructs occurred at $\sim 0.6 \mathrm{mg} / \mathrm{mL}$ with the circular solutions and earlier with the linear solutions $(\sim 0.3 \mathrm{mg} / \mathrm{mL}$ for $C-L$ and $\sim 0.4 \mathrm{mg} /$ $\mathrm{mL}$ for $L-L$ ). This finding was quite unexpected for the $C-L$ system. Constraint release is the only presently existing theory that can explain our finding of strongly hindered $C-L$ diffusion, yet $D \sim L^{-4}$ predicted for constraint release ${ }^{12}$ was not observed. Instead, the scaling was close to that predicted for reptation $(D$ $\sim L^{-2}$ ). This result agrees qualitatively with predictions for relaxed circular DNA diffusing in a gel, ${ }^{34}$ in which DNA was proposed to move around obstacles via finger-shaped loops (akin to the unpinned configurations in Klein's model). However, this agreement is perhaps only fortuitous as this model neglected pinned configurations, which would presumably be important in an entangled polymer solution.

Our results suggest that either the predicted scaling of $D$ with $L$ for constraint release is inaccurate or that a different diffusive process slower than reptation may be operating. Our finding of length scaling for $D_{C-C}, D_{C-L}$, and $D_{L-C}$ consistent with reptation is consistent with previous findings of studies of polystyrene by forward recoil spectrometry, ${ }^{24}$ but their findings of $D_{C-C} \cong D_{C-L}$ and $D_{L-C} \cong D_{L-L}$, differ from ours. Our finding of $D_{L-L}>D_{C-L}$ is qualitatively consistent with one study of polystyrene by forward recoil spectrometry, ${ }^{13}$ but a higher scaling exponent $(v=3.2-4.8)$ for $D_{C-L}$ was found in that study. While similar scaling of $D$ with $L$ was found for all topological cases, there was a dramatic difference in the magnitudes of $D$. While the $45 \mathrm{kbp}$ DNA diffused $\sim 4$ times slower than the $5.9 \mathrm{kbp}$ DNA at $0.1 \mathrm{mg} / \mathrm{mL}$ in both the $C-C$ and $C-L$ cases, it diffused $\sim 20$ times slower at $1.0 \mathrm{mg} / \mathrm{mL}$ in the $C-C$ case vs $\sim 1000$ times slower in the $C-L$ case. This finding was surprising, as the only previous experiment to compare $C-C$ and $C-L$ (in melts) had found $D_{C-C} \cong D_{C-L} \cdot{ }^{24}$

Direct comparisons with previous studies of synthetic polymer melts may also be complicated by inherent physical differences between different types of synthetic polymers as well as DNA. In certain situations, a concentration or molecular weight dependent monomeric friction coefficient is thought to contribute to the diffusion coefficient, in addition to Rouse, reptation, and constraint release dynamics. In particular, chain-end free volume effects can lead to a monomeric friction coefficient dependent on molecular length for certain linear chains. However, such chain-end effects have only been found to contribute significantly to diffusion for relatively short, rigid chains, ${ }^{23}$ whereas they have been found to be negligible for poly(ethylene oxide $)^{25}$ and PDMS chains above $N \cong 14$ (where $N$ is the number of bonds). ${ }^{23}$ Thus, our results for DNA of $\sim 40-300$ persistence lengths are not expected to be affected by such effects. Further, previous studies have shown that long DNA behave as semiflexible polymers, and thus free volume contributions should be negligible. ${ }^{19,31,35}$ Circular polymers are not subject to chainend effects, as they have no ends, but packing density effects can increase their diffusion rates in certain cases. In particular, small (low molecular weight) rings are likely to form flat disklike structures that can pack more efficiently than the ellipsoid configurations of larger rings and linear chains. ${ }^{23}$ However, this effect has been shown ${ }^{23}$ to be negligible for large rings $(N>20)$ such as those studied in our experiments.

\section{Conclusions}

We have shown that molecular topology can have an extremely strong effect on the diffusion of entangled polymers. This is the first study to systematically examine the four possible topological combinations with linear and circular molecules. Strong effects of topology were only apparent above a certain length and concentration. With the 5.9 and $11.1 \mathrm{kbp}$ molecules the results were largely insensitive to topology. We observed concentration scaling exponents of $\sim 0.5$ over most of the concentration range, only approaching 1.75 at the highest concentrations. $D \sim L^{-1}$ scaling was evident over most of the length range. Our interpretation is that the 5.9 and $11.1 \mathrm{kbp}$ molecules have lengths below the threshold for strong entanglement effects. With the 25 and $45 \mathrm{kbp}$ DNA the normalized $D$ values in the $L-C$ and $C-C$ cases fall approximately on the same curve, indicating that tracer topology has little effect in solutions of circular polymers. Both cases exhibit scaling close to that predicted by reptation ( $D \sim C^{-1.75}, D \sim L^{-2}$ ), supporting the conjecture that constraint release is negligible when the matrix is circular. ${ }^{11}$ Because circular molecules are more compact than linear molecules, it has been argued that circles would form entanglements less effectively. ${ }^{14}$ However, while we found $D_{L-L} \ll D_{L-C}$, the concentration threshold $C_{\mathrm{e}}$ appeared to be insensitive to topology. In the linear DNA solutions the tracer topology played a critical role. While $D_{C-L}$ and $D_{L-L}$ behaved similarly at low concentration, $D_{C-L}$ decreased dramatically, starting at $\sim 4 C^{*}$. Thus, a mechanism much slower than reptation must be operating in the $C-L$ case. Constraint release is the only model presently available to explain such slow diffusion; however, the measured scaling of $D$ with $L$ is weaker than that predicted for constraint release.

Acknowledgment. This research was supported by the Petroleum Research Fund of the American Chemical Society, a Sloan Fellowship to D.E.S., and a National Science Foundation Graduate Research Fellowship to R.M.R. 
Supporting Information Available: Table of contour lengths, number of persistence lengths, radii of gyration, and overlap concentrations for linear and relaxed circular DNA molecules. This material is available free of charge via the Internet at http://pubs.acs.org.

\section{References and Notes}

(1) McLeish, T. C. B. Adv. Phys. 2002, 51, 1379-1527.

(2) deGennes, P. G. Scaling Concepts in Polymer Physics; Cornell University Press: Ithaca, NY, 1979; p 324.

(3) Doi, M.; Edwards, S. F. The Theory of Polymer Dynamics; Oxford University Press: Oxford, 1986.

(4) Perkins, T. T.; Smith, D. E.; Chu, S. Science 1994, 264, 819-822.

(5) Smith, D. E.; Perkins, T. T.; Chu, S. Phys. Rev. Lett. 1995, 75, 41464149.

(6) von Meerwall, E. D.; Amis, E. J.; Ferry, J. D. Macromolecules 1985, $18,260-266$

(7) Amis, E. J.; Han, C. C. Polymer 1982, 23, 1403-1406.

(8) von Meerwall, E.; Grigsby, J.; Tomich, D.; Vanantwerp, R. J. Polym. Sci., Part B: Polym. Phys. 1982, 20, 1037-1053.

(9) Kolinski, A.; Skolnick, J.; Yaris, R. J. Chem. Phys. 1987, 86, 71647173.

(10) Kreer, T.; Baschnagel, J.; Muller, M.; Binder, K. Macromolecules 2001, 34, 1105-1117.

(11) Klein, J. Macromolecules 1986, 19, 105-118.

(12) Graessley, W. W. Adv. Polym. Sci. 1982, 47, 67-117.

(13) Mills, P. J.; Mayer, J. W.; Kramer, E. J.; Hadziioannou, G.; Lutz, P.; Strazielle, C.; Rempp, P.; Kovacs, A. J. Macromolecules 1987, 20 $513-518$.

(14) Brown, S.; Szamel, G. J. Chem. Phys. 1998, 109, 6184-6192.

(15) Muller, M.; Wittmer, J. P.; Cates, M. E. Phys. Rev. E 1996, 53, 50635074.

(16) Muller, M.; Wittmer, J. P.; Cates, M. E. Phys. Rev. E 2000, 61, 40784089.

(17) Ozisik, R.; Doruker, P.; Mattice, W. L.; von Meerwall, E. D. Comput. Theor. Polym. Sci. 2000, 10, 411-418.

(18) Ozisik, R.; von Meerwall, E. D.; Mattice, W. L. Polymer 2002, 43, 629-635.
(19) Cosgrove, T.; Griffiths, P. C.; Hollingshurst, J.; Richards, R. D. C.; Semlyen, J. A. Macromolecules 1992, 25, 6761-6764.

(20) Cosgrove, T.; Turner, M. J.; Griffiths, P. C.; Hollingshurst, J.; Shenton, M. J.; Semlyen, J. A. Polymer 1996, 37, 1535-1540.

(21) von Meerwall, E.; Ozisik, R.; Mattice, W. L.; Pfister, P. M. J. Chem. Phys. 2003, 118, 3867-3873.

(22) McKenna, G. B.; Hadziioannou, G.; Lutz, P.; Hild, G.; Strazielle, C.; Straupe, C.; Rempp, P.; Kovacs, A. J. Macromolecules 1987, 20, 498512.

(23) Orrah, D. J.; Semlyen, J. A.; Rossmurphy, S. B. Polymer 1988, 29, $1452-1454$.

(24) Tead, S. F.; Kramer, E. J.; Hadziioannou, G.; Antonietti, M.; Sillescu, H.; Lutz, P.; Strazielle, C. Macromolecules 1992, 25, 3942-3947.

(25) Griffiths, P. C.; Stilbs, P.; Yu, G. E.; Booth, C. J. Phys. Chem. 1995, 99, 16752-16756.

(26) Pecora, R. Science 1991, 251, 893-898.

(27) Rickgauer, J. P.; Smith, D. E. Single-Molecule Studies of DNA. In Soft Matter: Scattering, Imaging and Manipulation; Pecora, R. Borsali, R., Eds.; Kluwer: Dordrecht, in press.

(28) Shaqfeh, E. S. G. J. Non-Newtonian Fluid Mech. 2005, 130, 1-28.

(29) Teixeira, R.; Shaqfeh, E. S. G.; Chu, S. Macromolecules, in press.

(30) Laib, S.; Robertson, R. M.; Smith, D. E. Macromolecules 2006, 39, $4115-4119$.

(31) Robertson, R. M.; Laib, S.; Smith, D. E. Proc. Natl. Acad. Sci. U.S.A. 2006, 103, 7310-7314.

(32) Smith, D. E.; Perkins, T. T.; Chu, S. Macromolecules 1996, 29, 13721373.

(33) Because $D_{0}$ and thus $R_{\mathrm{G}}$ were measured for stained molecules which have a contour length $\sim 1.35$ times that of native DNA, ${ }^{32}$ the $R_{\mathrm{G}}$ values used in the equation for $C^{*}$ had to be scaled to the length of a native DNA molecule to achieve an accurate $C^{*}$ value for the unstained molecules This was done by using the relationship $R_{\mathrm{G} \text {,unstained }} / R_{\mathrm{G}, \text { stained }}$ $=\left(L_{\text {unstained }} / L_{\text {stained }}\right)^{v}$ where $v \cong 0.58$ is the scaling exponent measured in ref 31.

(34) Alon, U.; Mukamel, D. Phys. Rev. E 1997, 55, 1783-1793.

(35) Cosgrove, T.; Griffiths, P. C.; Webster, J. R. P. Polymer 1994, 35, 140-144.

MA070051H 